\title{
Clinical, Bacteriological, and Histopathological Findings of a Testicular Fibrosis in a 6-Year-Old Lusitano Stallion
}

\author{
A. Rocha, ${ }^{1,2}$ T. Guimarães, ${ }^{1,2}$ J. C. Duarte, ${ }^{3}$ C. Cosinha, ${ }^{3}$ V. T. A. Lopes, ${ }^{4}$ F. Faria, ${ }^{1}$ \\ I. Amorim, ${ }^{1,5}$ and F. Gärtner1,5 \\ ${ }^{1}$ Department of Immuno-Physiology and Farmacology (AR), Department of Veterinary Clinics (TG) and \\ Department of Pathology and Molecular Immunology (FF, IA and FG) of the Institute of Biomedical Sciences Abel Salazar (ICBAS), \\ University of Porto, Campus Agrário de Vairão, Rua Jorge Viterbo Ferreira no. 228, 4050-313 Porto, Portugal \\ ${ }^{2}$ Center for the Study of Animal Sciences (CECA), ICETA, University of Porto, Campus Agrário de Vairão, \\ Rua Padre Armando Quintas 7, 4485-661 Vairão, Portugal \\ ${ }^{3}$ LusoPecus, Rua da Fábrica-Azinhaga do Catalão, Loja 2-A-Porto Alto, 2135-000 Samora Correia, Portugal \\ ${ }^{4}$ EVP Lda, Rua Luís Derouet 27, Esquerdo 1, 1250-151 Lisbon, Portugal \\ ${ }^{5}$ Institute of Molecular Pathology and Immunology of the University of Porto (IPATIMUP), Rua Dr. Roberto Frias s/n, \\ 4200-465 Porto, Portugal
}

Correspondence should be addressed to F. Gärtner, fgartner@ipatimup.pt

Received 28 June 2012; Accepted 27 August 2012

Academic Editors: G. Sironi and D. M. Wong

Copyright ( 92012 A. Rocha et al. This is an open access article distributed under the Creative Commons Attribution License, which permits unrestricted use, distribution, and reproduction in any medium, provided the original work is properly cited.

\begin{abstract}
A 6-year-old Lusitano stallion was referred to our centre due to an enlarged left testicle. Anamnesis indicated that the stallion had a chronic hypertrophy of the left testicle, with no apparent ill effect on work (dressage training) or semen production. Prolonged use of anti-inflammatory drugs (NSAIDs) and antibiotics were probable. Upon examination of the animal, it was found that clinical signs were compatible with chronic testicular degeneration or fibrosis. Ultrasound scanning did not evidence the exuberant macroscopic lesions seen upon hemicastration of the left testicle, but it showed in the left spermatic cord a conspicuous absence of the typical hypoechogenic areas representing the pampiniform plexus. Swabbing of the penis, prepuce, and distal urethra resulted in the isolation of Rhodococcus equi and Corynebacterium spp. However, histopathological examination did not support infectious orchitis as cause of the lesions and no bacterial growth was obtained from swabbing of the parenchyma in the excised testicle. Histopathological findings were compatible with chronic orchitis with fibrosis and necrosis, probably secondary to ischemia of the testicular parenchyma. After hemi-castration, the stallion resumed semen production at acceptable levels.
\end{abstract}

\section{Introduction}

Testicular hypertrophy may have multiple causes, namely, testicular trauma, infectious orchitis, hydrocele, hematocele, neoplasia, or be secondary to torsion of the spermatic cord [1]. Publications on testicular pathology of the stallion in conjunction with clinical findings, ultrasound imaging, microbiological isolations, histopathological exams, and semen evaluation are scarce. In the present paper we describe the clinical, microbiological, and histopathological findings in a 6-year-old Lusitano stallion with an enlarged left testicle. Semen evaluation was assessed before and after hemi-castration, and possible etiology of the pathology is discussed.

\section{Case Presentation}

A 6-year-old Lusitano stallion was referred to our centre, due to an enlarged left testicle. When referred to us by a colleague, the stallion had been medicated with enrofloxacin, meloxicam and dexamethasone for the last $24 \mathrm{hrs}$. A previous use of anti-inflamatory drugs (NSAIDs) and antibiotics was probable. No colic-like signs had been noted in the stallion, but the owner referred to a transient "locomotion problem", several months before, during a horse show. The stallion was under dressage training and had been used intensively for semen collection and was reported as having very good libido, producing large volume ejaculates with a low concentration. 


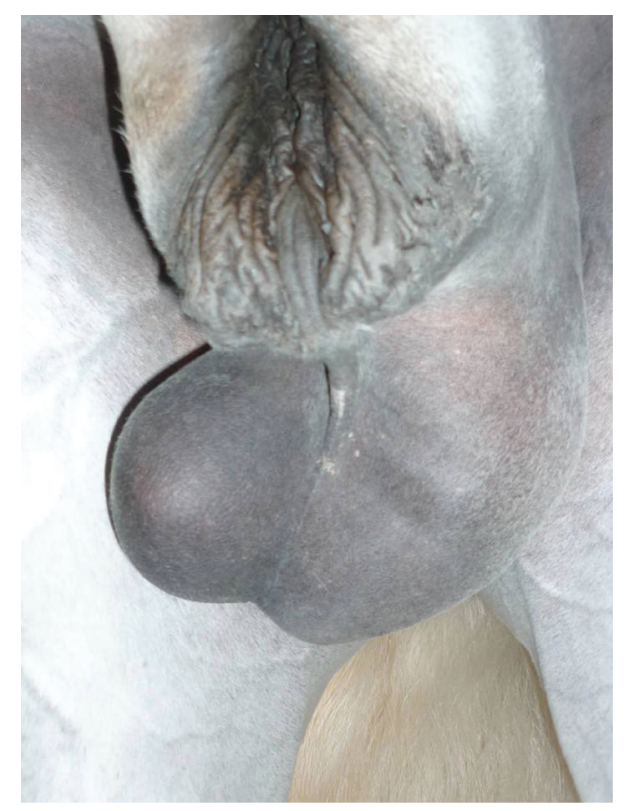

Figure 1: View of the testicles of the affected stallion with a greatly enlarged left testicle.

On arrival at our centre after a $4 \mathrm{hr}$ road trip, the stallion was eating normally (hay), did not limp, and at visual inspection displayed an obviously enlarged left scrotum, about two to three times the volume of the right normal-sized testicle (Figure 1). The rectal temperature was $37.6^{\circ} \mathrm{C}$. No other alterations were detected on physical exam. On hearing the mares vocalizing, the stallion immediately got a full erection. Semen was collected utilizing an artificial vagina (Missouri model) and a phantom as a mount. The stallion displayed very good libido and mounted and ejaculated with no signs of pain. Volume of the gel-free fraction of the ejaculate was $100 \mathrm{~mL}$, with a concentration of 85 million sperm cells/mL, a subjective motility of 30\% (optical microscopy, 200x), and linear motility assessed by computer-assisted sperm analysis (Integrated Sperm Analysis System, ISAS) of 20\%. Sperm cell morphology was assessed by optical microscopy $(\times 1000)$ in a Diff-Quik stained slide, counting 333 sperm cells. A total of $20.1 \%$ total abnormal forms were found, namely, $10.2 \%$ of the head (including acrosome), 8.1\% of the middle piece, and $1.8 \%$ of the tail. A few round, epithelial cells were seen as well as 3 monocytes.

Swabbing of the penis, prepuce, and distal urethra of the stallion was performed, and samples were sent for bacterial culture. A blood sample was collected by venipuncture of the jugular and sent for a complete blood analysis.

After semen collection the stallion was restrained in a stock and subjected to genital examination. At palpation, the skin of scrotum and spermatic cord was elastic and pliable without evidence of edema or dermal bruising. No edema was present in the prepuce either. The right scrotal contents felt normal, as well as the right spermatic cord, that measured $5 \mathrm{~cm}$ in its largest width. The head, body, and tail of the right epididymis were easily palpated and had a normal consistency. The left testicle felt very hard and tense and

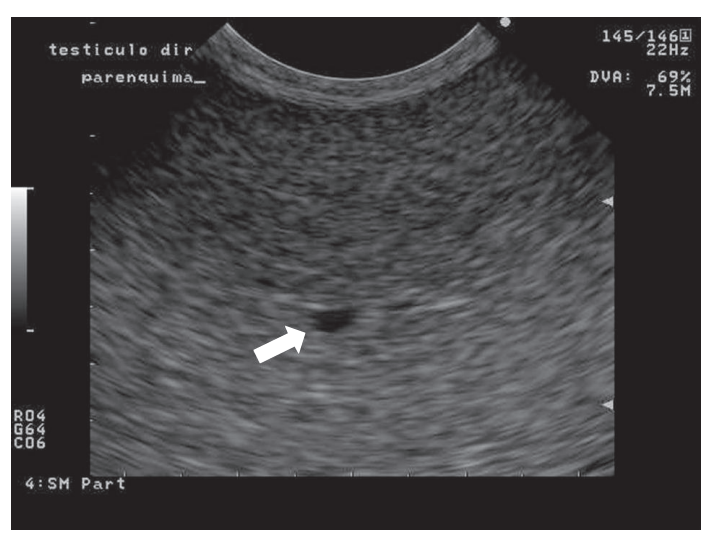

Figure 2: Ultrasonogram of the right testicle, showing homogenous parenchyma. White arrow indicates the central vein.

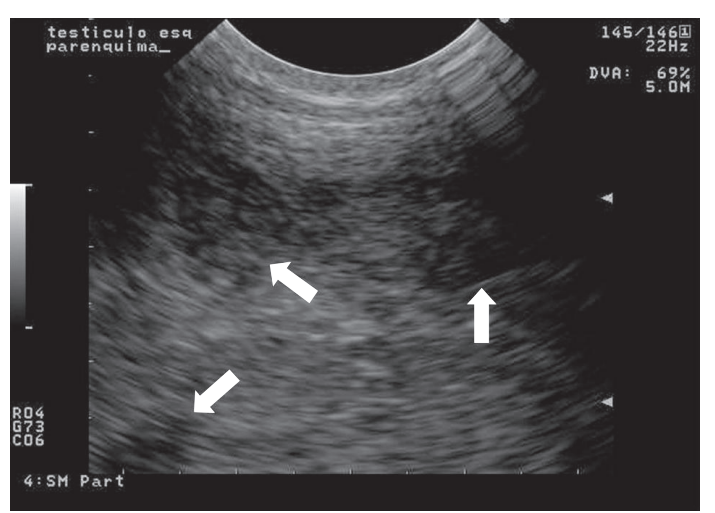

FIGURE 3: Ultrasonogram of the left testicle with several hypoechoic areas (white arrows).

had reduced mobility inside the scrotum. The animal did not display any sign of pain or discomfort at palpation, and scrotal temperature was not increased. The head and body of the left epididymis were difficult to individualize, but the tail was palpable in a normal position and location, attached to the caudal pole of the testicle. The left spermatic cord was very hard, with a width of $10 \mathrm{~cm}$ in the largest section.

Testicles, epididymides, and spermatic cord were scanned utilizing an Aloka ultrasound scanner model Prosound 2 with a $7.5 \mathrm{MHz}$ sector transducer. An abundant amount of ultrasound gel was used, but no stand-off pad was utilized. The parenchyma of the right testicle (Figure 2) presented a uniformly echogenic homogenous image, and the central vein could be visualized. No deviation from the expected ultrasound images was seen for the right head and cauda epididymis. The right spermatic cord presented the typical hypoechoic areas of the pampiniform plexus. No changes of echogenicity were seen in the left testicle and no scrotal edema was noticed, but the general texture of the testicular parenchyma was more granulose and coarse than the right testicle, with several anechoic, round areas with small $(50 \mathrm{~mm})$ diameter (Figure 3$)$. The central vein could not be visualized. The tail of the epididymis had normal echogenicity. The left spermatic cord did not show the typical 


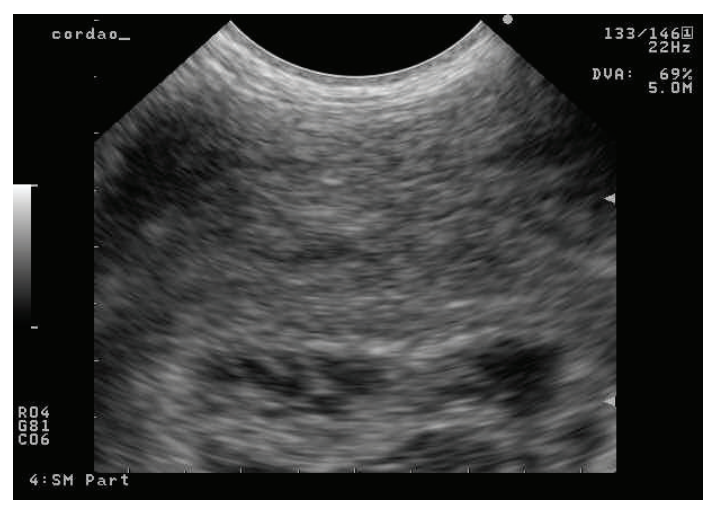

Figure 4: Ultrasonogram of the left spermatic cord without evidence of hypoechoic areas characteristic of the pampiniform plexus.

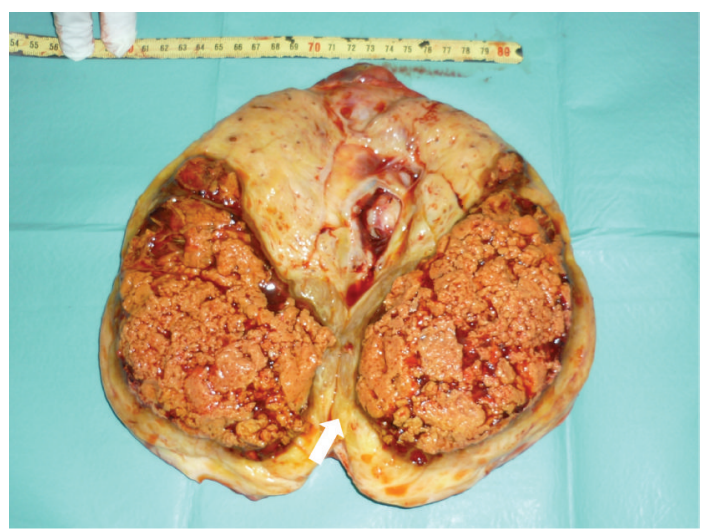

Figure 5: View of the excised testicle with the testicular parenchyma appearing as a soft mass. Note the increased thickness of the tunics (arrow).

hypoechoic areas characteristic of the pampiniform plexus. Instead, it seemed a rather solid, hyperechogenic area without much irrigation (Figure 4). Ultrasound scanning of the accessory sex glands, with the same ultrasound scanner using a linear $5.0 \mathrm{MHz}$ probe, did not evidence any alterations. A presumptive diagnosis of testicular degeneration or fibrosis of the left testicle was made, based on the findings of palpation and ultrasound examination. It was speculated that the testicular pathology could have been due to ischemia of the testicular parenchyma, possibly secondary to spermatic cord torsion. Given the possibility of formation of antisperm antibodies [2] that could be deleterious to the right, healthy testicle, immediate hemicastration was advised. The owner was renitent to that solution. Thus, the stallion was kept under NSAID and antibiotic therapy, without corticosteroids, and subjected to 15 -minute cold showers every $3 \mathrm{hrs,}$ in the affected testicle. As no improvements were seen in $48 \mathrm{hrs}$, the stallion was subjected to hemicastration of the left testicle, under general anesthesia. As soon as the excised testicle was opened, samples of four different areas of the parenchyma were collected aseptically, for bacterial isolation.

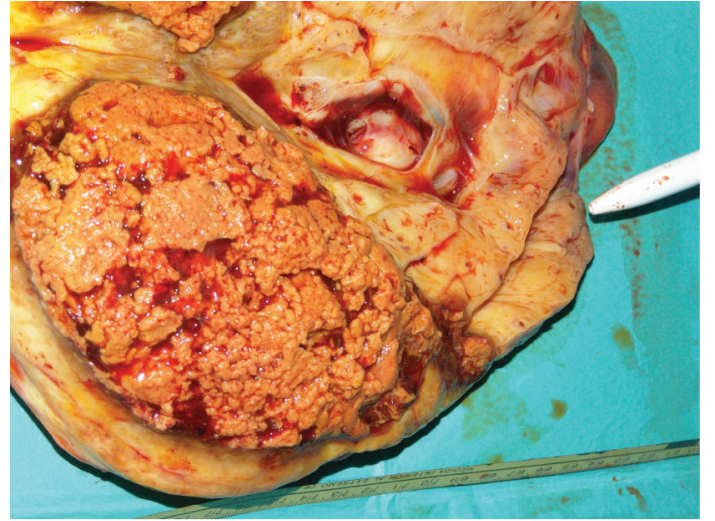

Figure 6: A view of the left epididymal head and body, appearing as a fibrotic dense tissues.

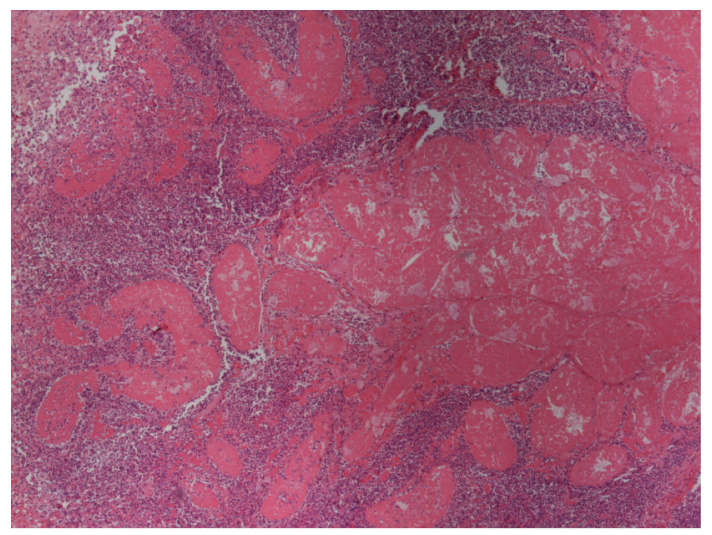

Figure 7: H\&E, 40x. Histologically, the lesion was composed of an extensive and central area of necrosis surrounded by large amount of diffuse mononuclear inflammatory infiltration.

The excised testicle was enlarged, with increased thickness of the tunics. The testicular parenchyma consisted of soft pink tissues forming cordons which string with easiness and presented a red liquefied appearance (Figure 5). The epididymis was hard at palpation and looked like a fibrotic dense tissue (Figure 6). Samples of the testicle were placed in formaldehyde and sent to the pathology lab for processing and histological analysis.

The microbiological isolation from the genital yielded pure cultures of Rhodococcus equi and Corynebacterium spp.. As no separated cultures were performed for the penis, prepuce, and distal urethra, it was not possible to precisely indicate where the growth of $R$. equi and Corynebacterium spp. occurred. No microbial growth was obtained from the testicular parenchyma samples.

At the histopathological exam, the testicular architecture was deeply modified, due to an extensive and central area of coagulation necrosis, with some neutrophils and cellular debris (Figure 7). At the periphery, moderate to severe amount of diffuse mononuclear inflammatory infiltration (rich in macrophages showing intense phagocytic activity, plasma cells, and lymphocytes) associated with 


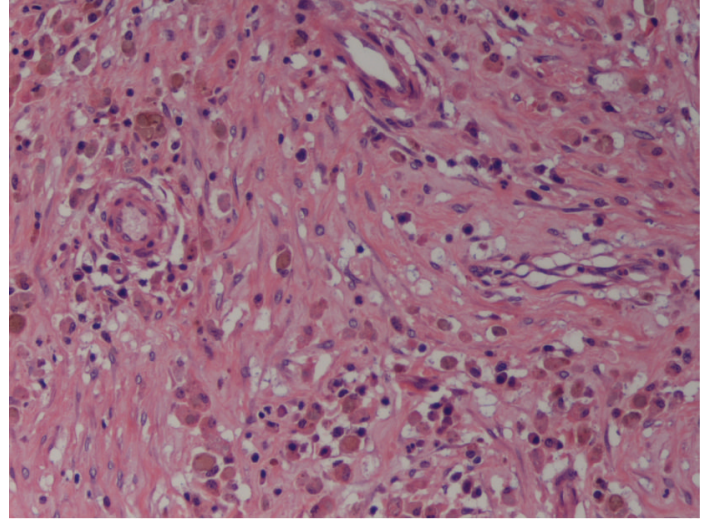

Figure 8: H\&E, 200x. The inflammatory infiltrate constited of macrophages showing intense phagocytic activity, plasma cells, and lymphocytes, with formation of an exuberant granulation tissue.

exuberant granulation tissue, rich in active fibroblasts and blood vessels, was noted (Figure 8). Also, multiple foci of lymphoplasmocitary infiltrate were observed. Most vessels evidenced degeneration of the walls, with accumulation of mucine-like material and cellular vacuolization (Figure 9). The epididymis was atrophic with very few sperm cells. Vital stains (periodic acid-Schiff and Gram) did not reveal any microorganisms. The histopathological findings were compatible with chronic orchitis, with severe fibrosis and necrosis. These findings, associated with the clinical signs, were compatible with lesions due to ischemia, possibly secondary to torsion of the spermatic cord $>180$ degrees.

All blood parameters were within normal range, including creatinine, aspartate aminotransferase (AST), gammaglutamyl transferase (GGT), and creatine kinase (CK).

Records from the semen collection centre (LusoPecus), just before the hemi-castration, showed that the progressive motility of the fresh semen in the 2 collected ejaculates was $60 \%$; a total of 60 straws with $100 \times 10^{6}$ sperm cells $/ \mathrm{mL}$ were frozen, with progressive motility at thawing of $40 \%$ and $30 \%$ for the first and second ejaculates, respectively. Four months after the hemi-castration, the stallion was collected 14 times during a period of 42 days, producing ejaculates with a subjective progressive motility ranging from $60 \%$ to $70 \%$, which resulted in the freezing of 20 to 60 straws with $100 \times 10^{6} \mathrm{sperm}$ cells $/ \mathrm{mL}$ per ejaculate, with a postthaw progressive motility varying from $35 \%$ to $40 \%$.

\section{Discussion}

Visual examination and palpation of the testicles were enough to clearly evidence a serious pathology of the left testicle, in this stallion. The use of ultrasonic imaging added some additional information that was not enough to establish a definitive diagnosis or to clearly show the existing total destruction of testicular parenchyma. However, ultrasonic images of the affected spermatic cord were useful to highlight the absence of images compatible with a functional pampiniform plexus. Extensive $\left(>180^{\circ}\right)$ torsion of the spermatic cord results in occlusion of the testicular

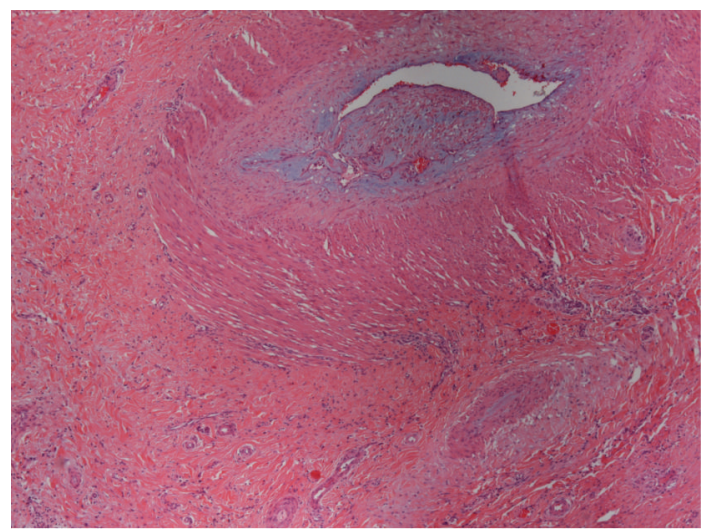

Figure 9: H\&E, 40x. Most vessels evidenced degeneration of the walls, with accumulation of mucin-like material and cellular vacuolization.

vasculature [2] and even $180^{\circ}$ torsions may cause retrograde blood flow and affect testicular function [3]. Thrombosis of the testicular artery may mimic the effects of testicular torsion, but is a rare condition [2]. Thus, we speculated that the lesions seen in the affected testicle could have been due to ischemia secondary to torsion of the spermatic cord, as no evidence of trauma was detected in the skin of the scrotum or of the spermatic cord.

Virulent strains Rhodococcus equi cause diseases in horses [4] including pyogranulomatous pneumonia in foals [5] and sporadically other lesions in several organs $[2,6]$. Rhodococcus equi has been isolated from the genital tract of mares [7, 8] and can cause abortion [7]. Upon receiving the microbiology results and considering the macroscopic aspect of the testicular parenchyma, the possibility of an ascending infection by Rhodococcus equi was considered. Together with the stallion of this case report, we collected during the reproductive season genital samples of 61 additional stallions, and the only positive result for $R$. equi was the reported case. Such a low prevalence is in agreement with results from Althouse et al. [9] that isolated $R$. equi only in 1 of 31 samples but differs from the findings of Spergser et al. [10] that isolated $R$. equi in 56 out of 116 stallions. The lack of isolation of bacteria from the parenchyma, associated with the histopathological findings, rules out an infectious origin of the testicular lesions observed.

In conclusion, visual inspection and testicular palpation of the scrotal contents were the best approach to reach a presumptive diagnosis, with ultrasonographic examination, particularly of the spermatic cord, adding some useful information. The real extent of the pathology was only seen after excision of the affected testicle. The most probable cause of the testicular pathology was ischemia of the testicular parenchyma, possibly after torsion of the spermatic cord. The isolation of pure colonies of Rhodococcus equi and Corynebacterium spp. from the genitalia may be due to opportunistic growth of these bacteria after prolonged use of antibiotics. Hemicastration of the affected testicle did not decrease the quality of the ejaculate or the freezability of the semen, compared to precastration levels. 


\section{Acknowledgments}

This work was partially financed by Projects PTDC/CVT/ 108456/2008 (FCT) and COMPETE: FCOMP-01-0124FEDER-009565. The authors appreciatively acknowledge Carla Mendonça, Luis Atayde, and Tiago Pereira for the castration of the stallion and Carla Miranda, Eliane Silva, and Gertrude Thompson for the microbiological isolations.

\section{References}

[1] W. Beard, "Abnormalities of the testicles," in Equine Reproduction, A. O. McKinnon, E. L. Squires, W. E. Vaala, and D. D. Varner, Eds., pp. 1161-1165, Wiley-Blackwell, 2nd edition, 2011.

[2] J. Schumacher and D. D. Varner, "Abnormalities of the spermatic cord," in Equine Reproduction, A. O. McKinnon, E. L. Squires, W. E. Vaala, and D. D. Varner, Eds., pp. 1145-1155, Wiley-Blackwell, 2nd edition, 2011.

[3] M. A. Pozor and S. M. McDonnell, "Color Doppler ultrasound evaluation of testicular blood flow in stallions," Theriogenology, vol. 61, no. 5, pp. 799-810, 2004.

[4] R. Wada, M. Kamada, T. Anzai et al., "Pathogenicity and virulence of Rhodococcus equi in foals following intratracheal challenge," Veterinary Microbiology, vol. 56, no. 3-4, pp. 301-312, 1997.

[5] D. L. Dungworth, "Rhodococcus (Corynebacterium) equïnfection," in Pathology of Domestic Animals, K. V. F. Jubb, P. C. Kennedy, and N. Palmer, Eds., vol. 2, pp. 652-655, Academic Press, 4th edition, 1993.

[6] M. C. Zink, J. A. Yager, and N. L. Smart, "Corynebacterium equi infections in horses, 1958-1984: a review of 131 cases," Canadian Veterinary Journal, vol. 27, pp. 213-217, 1986.

[7] L. Szeredi, T. Molnár, R. Glávits et al., "Two cases of equine abortion caused by Rhodococcus equi," Veterinary Pathology, vol. 43, no. 2, pp. 208-211, 2006.

[8] A. M. Bain, "Corynebacterium equi infections in the equine," Australian Veterinary Journal, vol. 39, pp. 116-121, 1963.

[9] G. C. Althouse, J. Skaife, and P. Loomis, "Prevalence and types of contaminant bacteria in extended, chilled equine semen," Animal Reproduction Science, vol. 121, pp. S224-S225, 2010.

[10] J. Spergser, C. Aurich, J. E. Aurich, and R. Rosengarten, "High prevalence of mycoplasmas in the genital tract of asymptomatic stallions in Austria," Veterinary Microbiology, vol. 87, no. 2, pp. 119-129, 2002. 

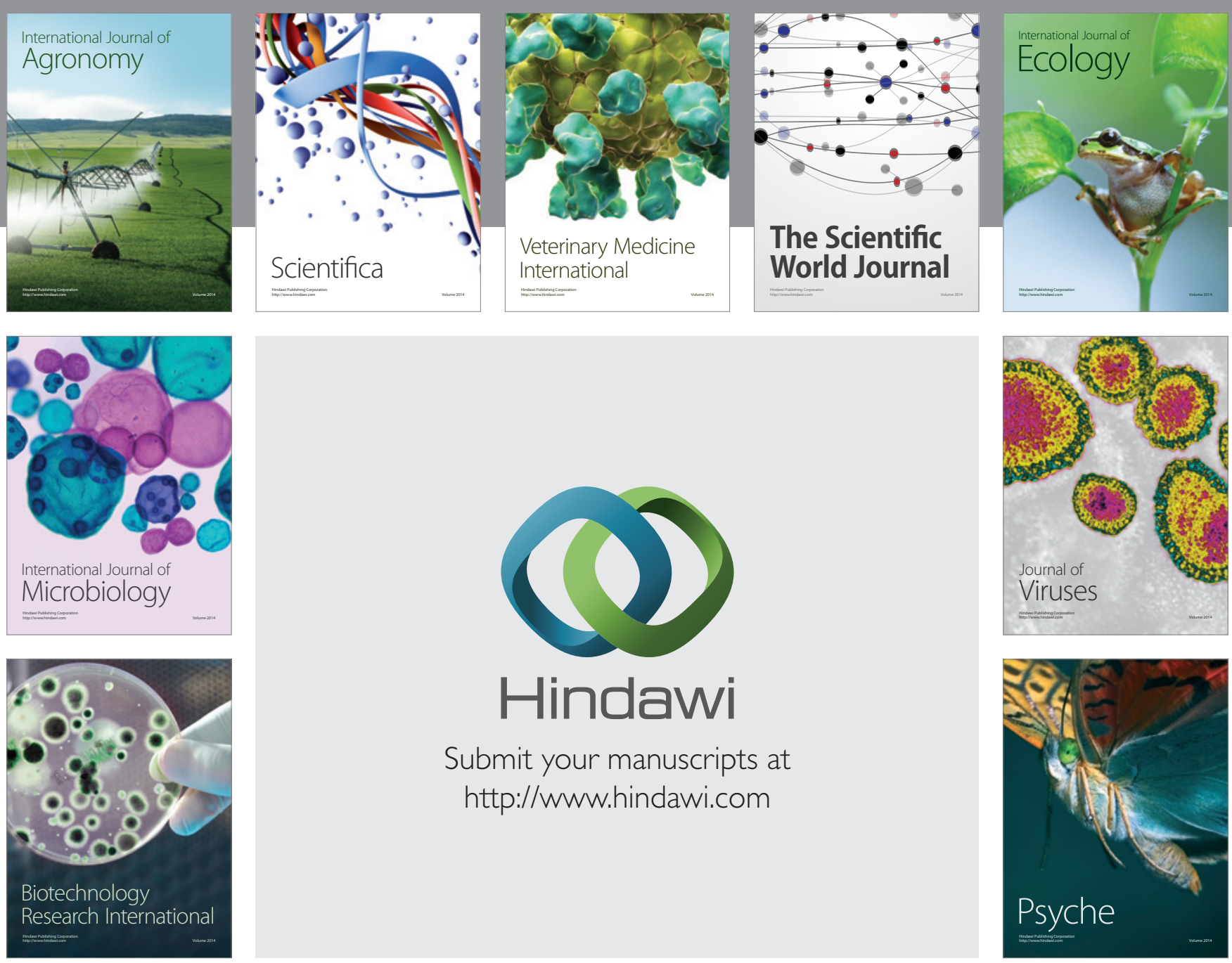

Submit your manuscripts at

http://www.hindawi.com
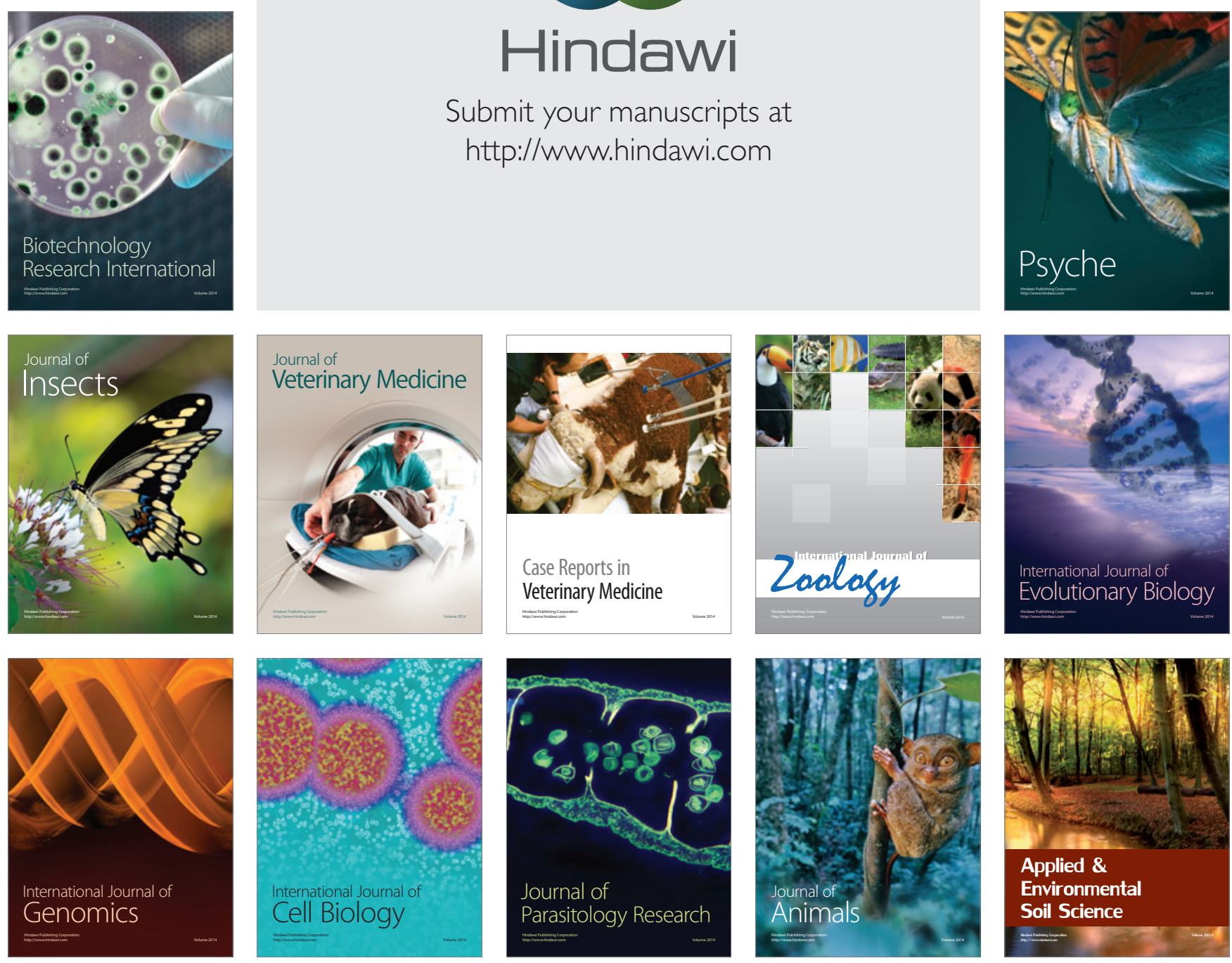